\section{TAKING A BITE OUT OF HEARING LOSS}

The SoundBite Hearing System from Sonitus Medical, which is placed in the ear canal and around the back teeth, is designed to help patients who are deaf in one ear regain their spatial hearing ability. Labelled 'the world's first non-surgical and removable hearing solution', the custom-made inserted hearing device requires no alteration to teeth.

Sounds picked up from a behind-the-ear microphone unit on the deaf side are wirelessly transmitted to the removable in-the-mouth (ITM) device, which then sends out imperceptible vibrations from the teeth through the skull bones using bone conduction, to the functioning cochlea on the hearing side. The ITM device can be made to fit around the upper left or right back teeth, but as the sound waves need a firm jaw connection for bone conduction, patients with removable dentures cannot wear the SoundBite.

Clinical research published in the journal Otology Et Neurotology (2011; 32: 437-443) has shown

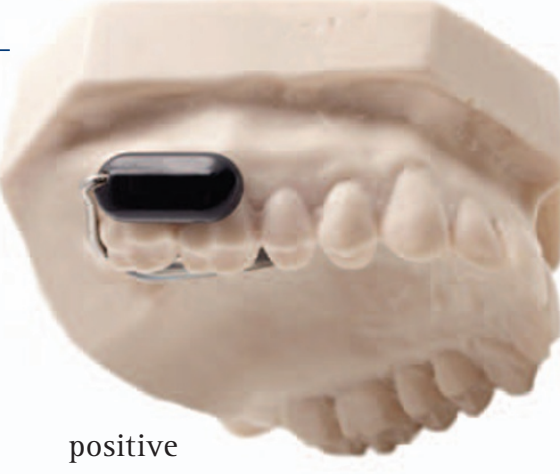

results for

the SoundBite, with participants suffering single-sided deafness noting a marked improvement in their hearing and no dental complications or discomfort.

Sonitus Medical intends to develop its SoundBite conductive technology for mixed hearing loss patients in the future. www. sonitusmedical.com/product/

\title{
DENTURES WITH A TIGHTER FIT THAT EXPOSE THE SENSES
}

A New Zealand based pharmaceutical company has developed a denture system that uses suction to hold the denture in place. UltraSuction denture users will never have to use denture adhesives again, or put up with the daily struggle to keep their dentures firmly in place.

This new style of advanced denture is proven to increase stability and offers an improved resistance to dislodging forces. It will be an optional extra for some patients but a necessity for others; either way the dentures will be a tighter fit.

It is also a fact that dentures get in the way of natural sense, be it taste or smell. With the use of UltraSuction, denture size is vastly reduced, thereby exposing more aural sense receptors. Furthermore, most upper dentures with this new system built in are horseshoe shaped, eliminating the need for the upper acrylic palate, which covers most of the natural palate of the mouth. This, in turn, exposes the patient's senses and allows a more natural feel.

UltraSuction dentures have been described as the step in between conventional dentures and dental implants.

Contact your lab today and ask about UltraSuction dentures, or contact the UK distributors for advice by emailing chrisb@abacus-dental.com.

\section{VITAL TRAINING FOR YOUR TEAM}

The British Dental Journal's sister publication Vital offers two hours of verifiable continuing professional development (CPD) per issue, or eight hours per year, specifically targeted at dental care professionals (DCPs).

The Vital verifiable CPD scheme is available to any DCP who would like to take part and offers CPD in the core topics recommended by the General Dental Council (GDC) over the five-year CPD cycle.

In the latest issue (summer 2012) there are CPD articles on medical emergencies and on how to manage patients with pacemakers. CPD multiple choice questions on these articles are available until just before the autumn issue is published in September 2012.

It is free to register on the Vital website and access all articles from 2003 to present at www. nature.com/vital. A subscription to Vital CPD can be purchased for individuals via the site, with one year (eight hours of CPD) costing $£ 30+$ VAT which equates to less than $£ 5$ per hour of verifiable CPD.
NINETY MINUTE CANCER DETECTION COURSE

Dental healthcare professionals have a key role to play in the early detection and treatment of oral cancer and can learn more through the 90 minute 'Oral Cancer; Prevention, Examination and Referral' course developed by Smile-on with the Department of Health, Dental Protection Ltd, KSS Deanery and the British Dental Health Foundation.

The course covers: current oral cancer epidemiology and latest research; the vital role of routine opportunistic screening in early detection; identification of suspicious lesions; appropriate referral procedures; effective and empathetic communication with patients; and understanding post referral outcomes.

Through raising awareness of risk factors such as smoking, alcohol abuse, poor diet, using chewing or smokeless tobacco and the sexually transmitted human papilloma virus (HPV), dental professionals will be able to offer appropriate advice, support and screening.

For more information call 02074008989 or email info@ smile-on.com.

Participants

can save or print off their CPD certificates directly from the site.

DCPs can also include reading past articles from the Vital archive in their general (non-verifiable) CPD.

www.nature.com/vitalcpd 